\title{
Model-Independent Bounding of the Supports of Boolean Formulae in Binary Data
}

\author{
Artur Bykowski ${ }^{1}$, Jouni K. Seppänen ${ }^{2}$, and Jaakko Hollmén ${ }^{2}$ \\ 1 LISI, INSA-Lyon, Bât. Blaise Pascal, 20, ave A. Einstein, \\ F-69621 Villeurbanne Cedex, France \\ Artur.Bykowski@insa-lyon.fr \\ 2 Helsinki University of Technology, Laboratory of Computer and \\ Information Science, P.O. Box 5400, 02015 HUT, Finland \\ \{Jouni.Seppanen, Jaakko.Hollmen\}@hut.fi
}

\begin{abstract}
Data mining algorithms such as the Apriori method for finding frequent sets in sparse binary data can be used for efficient computation of a large number of summaries from huge data sets. The collection of frequent sets gives a collection of marginal frequencies about the underlying data set. Sometimes, we would like to use a collection of such marginal frequencies instead of the entire data set (e.g. when the original data is inaccessible for confidentiality reasons) to compute other interesting summaries. Using combinatorial arguments, we may obtain tight upper and lower bounds on the values of inferred summaries. In this paper, we consider a class of summaries wider than frequent sets, namely that of frequencies of arbitrary Boolean formulae. Given frequencies of a number of any different Boolean formulae, we consider the problem of finding tight bounds on the frequency of another arbitrary formula. We give a general formulation of the problem of bounding formula frequencies given some background information, and show how the bounds can be obtained by solving a linear programming problem. We illustrate the accuracy of the bounds by giving empirical results on real data sets.
\end{abstract}

\section{Introduction}

Database management systems allow querying extensional data and intentional data. From the user's point of view, extensional data are the data explicitly input by the user into the system. Intentional data are not put in by the user-that information is derived from extensional data.

In inductive database management systems the intentional data are usually quite complex from various points of view and require a high computational effort to obtain. In case of typical patterns (frequent sets, association rules), the common problem is that the domain of patterns is prohibitively large and the inductive database management system cannot compute them all. The typical approach is to let the user guide the system to the interesting patterns interactively, e.g., through queries, limiting the search space to be considered.

Then, the question is if the result of one query could be reused at least partly for obtaining the next result as an alternative to re-computing the whole answer 
from extensional data. The following, more difficult question is whether there might be some summaries that the inductive database management system can gather off-line before the data mining process, to improve the on-line behavior of most mining processes. Typically, one would be interested in sufficient statistics, i.e., summaries that can be substituted for the whole extensional data to avoid repetitive probing of the selection predicate for all candidate patterns. On the other hand, we prefer gathering summaries that are known to be efficient to obtain.

In this paper we pinpoint how to take advantage in a particular context of a collection of summary queries that have been evaluated against the extensional data to bound the value of the evaluation functions of other queries. Providing bounds may be interesting when we have thresholds on the evaluation function, and a tight bound can enable us to make a correct decision about accepting or rejecting a pattern in the query answer. We focus on the context of reusing previous queries (without pre-selecting) and leave open the question of choosing beforehand which summaries should be used.

Several data mining algorithms can be used for efficient computation of a large number of summaries from data. Such methods include Apriori-type algorithms for finding frequent sets $\mathrm{AMS}^{+}$96] or episodes [MTV97] in binary or sequential data and methods for clustering large data sets [ZRL97. The summary information given by such algorithms can then be used as an efficient condensed representation of the data set. When the available summaries are orders of magnitude smaller than the data set itself (typical in case of huge data sets in a data mining context), it could be worth using them instead of the entire data set to compute other interesting summaries. Typically, the information contained in a collection of summaries will not be sufficient to compute the precise value of all other summaries, but at least bounds could be inferred. If the accuracy of the estimated result is not enough, the partial quantitative information (bounds) can be used to better optimize the query execution plan (of a query to the original data set).

An interesting fundamental question is: how much information about the underlying data set does a collection of summaries give? In this paper we consider this question in the setting of frequent sets for binary data. Information of frequencies of different itemsets can have strong implications for the frequencies of other itemsets. For example, if we know that $f(A B)=f(A)$, then we know that $f(X A)=f(X A B)$ for any set $X$, a result that has been shown to be surprisingly useful in the context of so-called closures [PBTL99] and free sets BBR00BR01. Also, we know that the frequency $f(X)$ of an itemset $X$ is bounded from above by the minimum support $f(Y)$ of a subset $Y$ of $X$.

More generally, if we possess the information about the frequencies of some Boolean formulae (frequent itemsets being a particular case), the frequency of any other Boolean formula can be inferred, to some extent. The main question we pose in this paper is how we could efficiently construct upper and lower bounds

\footnotetext{
${ }^{1}$ Here we denote by $f(A B)$ the frequency of the itemset $\{A, B\}$.
} 
for the frequencies of any Boolean formula, given the existing information. We show that this formula bounding problem can in fact be solved by transforming the question into a linear program and solving that problem. In the worst case the transformation leads to a program of exponential size, but we also give empirical results showing that the transformation in many cases is an efficient one.

The paper is organized as follows. In Section 2 we define the basic notions and the support bounding task. Section 3 gives the solution, and Section 4 describes the empirical results. Finally, in Section 5, we summarize the paper and discuss open problems.

\section{Problem: Support Queries in Databases}

The problem we want to solve involves Boolean queries on binary relational databases. In order to present the problem in an exact way, we first make some formal definitions.

Definition 1. A relation $r$ over the finite attribute set $X$ is a finite multiset of tuples, subsets of $X$. The degree of $r$ is the cardinality of $X$, and the size of $r$ is the (multiset) cardinality $|r|$ of $r$. The set $X$ is called the schema of $r$.

In contrast to ordinary relational databases, we deal with binary data only. This allows a convenient notational shortcut: for example, instead of the tuple $(0,1,1,0,1)$ over the attributes $A, B, C, D, E$, we can talk about the tuple $\{B, C, E\}$. Also, a Boolean query such as " $(A=1$ and $B=1)$ or $(C=1$ and $D=0)$ " can be written as " $A$ and $B)$ or $(C$ and not $D)$ ", or, in the algebraic notation, $A B+C \bar{D}$. The syntax and semantics of such queries are defined next.

Definition 2. A Boolean formula over the attribute set $X$ is one of:

1. $\top$ (the true constant),

2. A for some attribute $A \in X$ (an atom),

3. $(\neg \phi)$ for some Boolean formula $\phi$ over $X$ (a negation),

4. $(\phi \psi)$ for some Boolean formulae $\phi, \psi$ over $X$ (a conjunction),

5. $(\phi+\psi)$ for some Boolean formulae $\phi, \psi$ over $X$ (a disjunction).

We omit parentheses when there is no danger of ambiguity. Furthermore, the negation operator $\neg$ always binds to the shortest following subformula, and conjunction binds tighter than disjunction. In the case of negated atoms, we also write $\bar{A}$ for $\neg A$. Thus, $\bar{A} B C+A(B+\bar{C})$ means $(((\neg A) B) C)+(A(B+$ $(\neg C)))$ ). These conventions leave it ambiguous in which direction conjunction and disjunction associate, but in fact all readings of an ambiguous formula have equivalent semantics by the following definition.

Definition 3. Given a tuple $t \in r$, we define the truth value of all Boolean formulae over the schema of $r$ as follows. 
1. $[\top]_{t}=1$,

2. $[A]_{t}=1$ if $A \in t,[A]_{t}=0$ if $A \notin t$,

3. $[\neg \phi]_{t}=1-[\phi]_{t}$,

4. $[(\phi \psi)]_{t}=[\phi]_{t}[\psi]_{t}$,

5. $[(\phi+\psi)]_{t}=[\phi]_{t}+[\psi]_{t}-[\phi]_{t}[\psi]_{t}$.

Two formulae $\phi$ and $\psi$ are equivalent, if they always have the same truth value on the same tuple. That all readings of an ambiguous formula such as $\phi \psi \theta$ are equivalent is a standard result in propositional logic. Different in our problem is that we extend the semantics to whole relations.

Definition 4. Let $r$ be a relation and $\phi$ a Boolean formula over a common schema. Then the support of $\phi$ in $r$ is the proportion of tuples in $r$ for which $\phi$ is true,

$$
[\phi]_{r}=|r|^{-1} \sum_{t \in r}[\phi]_{t} .
$$

We write $[\phi]$ when the relation is clear from the context.

The data mining literature contains a wealth of material on itemsets, sets of attributes. After Boolean formulae have been defined, it is easy to give semantics to itemsets as simple conjunctions.

Definition 5. Let $X$ be a relation schema. A subset of $X$ is an itemset, and it is identified with the conjunction of all its elements.

The name "itemset" originated in association rule mining, whose traditional application is market-basket data: the attributes are items offered for sale at a supermarket, and the tuples are customer transactions. It turns out that to find association rules that are in a certain sense interesting, it suffices to compute all itemsets whose support exceeds a threshold. This is usually done by a breadth-first search algorithm called Apriori $\mathrm{AMS}^{+} 96$, but several variations have been proposed. For example, depth-first search can be performed using FPtrees HPY00, and a sampling approach can avoid database scans [Toi96]. An active area of research is mining not all frequent itemsets but only an interesting subfamily; see e.g. GZ01CG02 PBTL99 BBR00BR01.

As an example, Table 1 shows a small binary database. We have e.g. $[A]_{t}=$ $[A]_{u}=[A B]_{t}=[A C]_{u}=1$ and $[A]_{v}=[B]_{u}=[A B]_{u}=[A C]_{v}=0$, and over the whole database $[A]_{r}=2 / 3,[B]_{r}=1 / 3,[C]_{r}=1,[A C]_{r}=2 / 3$, and $[A B C]_{r}=$ $1 / 3$. If the frequency threshold is $1 / 2$, the frequent itemsets are $A, C, A C$, and trivially the empty set, which corresponds to $T$.

Table 1. An example database $r$

\begin{tabular}{cccc}
\hline Tuple & $A$ & $B$ & $C$ \\
\hline$t$ & 1 & 1 & 1 \\
$u$ & 1 & 0 & 1 \\
$v$ & 0 & 0 & 1 \\
\hline
\end{tabular}


We now come to the formula bounding problem. Given are a set $\Phi$ of Boolean formulae over a schema $X$, and their supports in an unknown relation $r$. The desired result is the support of another formula $\psi$. This support is sometimes completely determined by the givens, but this is rare; in general we want the set of all possible supports. As it turns out, the minimum and maximum support determine this set completely, and we can allow minima and maxima also as inputs. We denote by $\operatorname{Int}_{\mathbb{Q}}(0,1)$ the set of closed intervals $[a, b]$ of rational numbers where $0 \leq a \leq b \leq 1$.

Definition 6. The formula bounding task $\operatorname{Bound}(X, \Phi, f, \psi)$ has the following inputs: a relation schema $X$, a set $\Phi$ of Boolean formulae over $X$, a function $f$ from $\Phi$ to $\operatorname{Int}_{\mathbb{Q}}(0,1)$, and a Boolean formula $\psi$ over $X$. The solution of the task is the smallest set $I \subseteq[0,1]$ such that $[\psi]_{r} \in I$ for all relations $r$ over $X$ fulfilling the constraint $[\phi]_{r} \in f(\phi)$ for all $\phi \in \Phi$.

In other words, we want a sound and complete inference procedure for the support bounds of Boolean formulae. We call a procedure sound if its result $I$ rules out no possible solutions: for $q \notin I$, there should be no relation $r$ fulfilling the constraints defined by $f$ such that $[\psi]_{r}=q$. Conversely, the set $I$ returned by a complete procedure is such that every solution $q \in I$ is realizable in some relation fulfilling the constraints. (A trivially complete but non-sound procedure returns $I=\emptyset$ for all inputs; the similar sound but non-complete procedure always returns $I=[0,1]$.) The problem is NP-hard, since it requires solving the satisfiability of $\psi$.

The following lemma shows that it suffices to find upper and lower bounds for the numbers in $I$. Thus, the task has a closure property: the output is in the same form as each element of the input.

Lemma 1. If the solution $I$ of $\operatorname{Bound}(X, \Phi, f, \psi)$ is nonempty, then $I$ is an interval of rational numbers.

Proof. We must prove that given any three rationals $P, W, Q \in[0,1]$ with $P<$ $W<Q$ and $P, Q \in I$, also $W \in I$. Since $W$ lies between $P$ and $Q$, there is a rational number $Z \in(0,1)$ such that $W=Z P+(1-Z) Q$. Since $P, Q \in I$, there exist relations $p, q$ fulfilling the constraints of the bounding problem such that $[\psi]_{p}=P$ and $[\psi]_{q}=Q$. We will construct a relation $w$ for which the support of all formulae $\theta$ over $X$ is $[\theta]_{w}=Z[\theta]_{p}+(1-Z)[\theta]_{q}$. Since $[\theta]_{w}$ lies between the numbers $[\theta]_{p}$ and $[\theta]_{q}$, every inequality constraint $[\phi]_{w} \in f(\phi)$ will be satisfied. Further, $[\psi]_{w}=W$, as required.

To construct the relation $w$, we would like to take $Z /|p|$ copies of all tuples in $p$ and $(1-Z) /|q|$ copies of all tuples in $q$. This is impossible in the general case, but if we multiply the numbers $Z /|p|$ and $(1-Z) /|q|$ by the least common multiple of their denominators, we can replace the numbers by integer multiples. It is then easy to check that $[\theta]_{w}=Z[\theta]_{p}+(1-Z)[\theta]_{q}$ for all formulae $\theta$. 


\section{Solving the Bounding Task by Linear Programming}

In this section, we describe a solution to the Bound task of Definition 6. The solution is based on linear programming, and it is both sound and complete.

\subsection{Change of Variables}

Several kinds of equalities and inequalities hold in all relations. For example, $[A+B]=[A]+[B]-[A B]$ by the combinatorial inclusion-exclusion principle, and $0 \leq[A B] \leq[A] \leq 1$ by the anti-monotonicity of support. A procedure for the Bound task has to incorporate all results of this type.

Let us analyze how these results could be proved. The middle inequality follows from the observation that $[A]=[A B]+[A \bar{B}]$ and that the support of $[A \bar{B}]$ lies in the interval $[0,1]$. A similar idea gives a proof of the inclusion-exclusion formula:

$$
\begin{aligned}
{[A+B] } & =[A B]+[A \bar{B}]+[\bar{A} B] \\
& =([A B]+[A \bar{B}])+([A B]+[\bar{A} B])-[A B]=[A]+[B]-[A B] .
\end{aligned}
$$

This suggests that a change of variables can make the needed results simpler to prove. To that end, we make the following definitions.

Definition 7. Given an attribute $A \in X$, the positive literal based on $A$ is the Boolean formula $A$, and the negative literal based on $A$ is the Boolean formula $\bar{A}$. $A$ literal based on $A$ is either the positive literal or the negative literal based on $A$.

Definition 8. A clause over the attribute set $X$ is a conjunction of zero or more literals based on different attributes.

Our definition of a clause allows an attribute to appear at most once, in either a negative or a positive literal. For example, $B \bar{C} \bar{E}$ is a clause over the set $\{A, B, C, D, E\}$, but $B \bar{B} \bar{E}$ and $B B \bar{E}$ are not. The true constant $\top$ is a clause as the degenerate case of zero literals.

Definition 9. A full clause over the attribute set $X$ is a clause with exactly $|X|$ literals.

In a full clause each attribute appears exactly once, either as a negative or a positive literal. For example, the conjunction $A B \bar{C} D \bar{E}$ is a full clause over the set $\{A, B, C, D, E\}$, whereas $B \bar{C} \bar{E}$ is not. In the language of propositional logic, a full clause fully describes a model over the given attribute set.

Full clauses are important for two reasons. First, there is a natural correspondence between relations and assignments of supports to full clauses. Given a relation $r$, any full clause $\theta$ over the schema of $r$ is satisfied by some nonnegative integral number of identical tuples in $r$. Conversely, given an assignment of nonnegative rational supports for all full clauses summing up to 1 , it is simple to construct a relation giving rise to these supports.

The second reason is that all formulae can be decomposed into full clauses (for formulae corresponding to typical queries it is easy). We record this in the following two propositions. 
Proposition 1. Every Boolean formula over an attribute set $X$ can be equivalently written as a disjunction $C_{1}+C_{2}+\cdots+C_{p}$ of distinct full clauses $C_{i}$. We call this the full disjunctive normal form.

Proposition 2. The support of any Boolean formula $\phi$ over an attribute set $X$ can be written as a sum of supports of distinct full clauses. That is, there is a set of full clauses $C_{1}, C_{2}, \ldots, C_{p}$ such that $[\phi]_{r}=\left[C_{1}\right]_{r}+\left[C_{2}\right]_{r}+\cdots+\left[C_{p}\right]_{r}$ for any relation $r$ over $X$.

These results enable us to untangle the complex interrelations of formulae. The supports of distinct full clauses are independent of each other 2 , so any distribution of nonnegative supports for full clauses corresponds to a possible relation. Where the support of a Boolean formula appears in a constraint equality or inequality, we can invoke Proposition 2 to replace it by a sum of the supports of the corresponding full clauses. This amounts to a linear change of variables.

As an example, we consider an instance of $\operatorname{Bound}(X, \Phi, f, \psi)$ with $X=$ $\{A, B\}, \Phi=\{\top, A, B, A B\}$, and $\psi=A B$. After the change of variables, we have the system depicted in Table 2 which we should solve for $[A B]$. We have the additional information that $0 \leq\left[\theta_{i}\right] \leq 1$ for all formulae $\theta_{i}$, but we need not worry about the inclusion-exclusion principle or similar rules. We continue this example at the end of Section 3.2

Table 2. Example bounding task with decomposition into full clauses

\begin{tabular}{rllll}
\hline & $A B$ & $A \bar{B} \bar{A} B \bar{A} \bar{B}$ \\
\hline$[\top]=1.0$ & $\times$ & $\times$ & $\times$ & $\times$ \\
{$[A]=0.6$} & $\times$ & $\times$ & & \\
{$[B]=0.7$} & $\times$ & & $\times$ & \\
{$[A B] \in[0,0.5]$} & $\times$ & & & \\
\hline
\end{tabular}

\subsection{Linear Programming}

We now turn to the classic optimization problem called linear programming. We only describe it briefly; see, e.g., Chapter 21 in Kre93 for a good introduction to the subject, or the Linear Programming FAQ 3 for a comprehensive list of references. For the computational complexity of linear programming, see e.g. MSW96; briefly, common algorithms such as Simplex tend to be useful in practice although they have worst-case exponential complexity, but more sophisticated algorithms such as Karmarkar's algorithm Kar84 achieve lower complexity.

${ }^{2}$ With the restriction that the supports of all full clauses sum up to 1 ; but this gives only a scaling factor.

3 http://www-unix.mcs.anl.gov/otc/Guide/faq/linear-programming-faq.html 
Definition 10. The linear programming problem $\operatorname{LP}(\mathcal{A}, \mathcal{B}, \mathcal{C})$ comprises an $m \times$ $n$ matrix $\mathcal{A}$, an $m$-element column vector $(m \times 1$ matrix $) \mathcal{B}$, and an $n$-element row vector $(1 \times n$ matrix $) \mathcal{C}$. The solution of the problem is the vector $\boldsymbol{x}$ that minimizes the scalar value $\mathcal{C} \boldsymbol{x}$ subject to the restrictions $\mathcal{A} \boldsymbol{x} \leq \mathcal{B}, \boldsymbol{x} \geq \mathbf{0}$. We also denote by $\operatorname{LP}^{\prime}(\mathcal{A}, \mathcal{B}, \mathcal{C})$ the problem that is otherwise similar but where the first restriction is replaced by $\mathcal{A} \boldsymbol{x}=\mathcal{B}$.

The matrix $\mathcal{C}$ is said to express the objective function, and $\mathcal{A}$ and $\mathcal{B}$ state the constraints of the problem.

The problems $\operatorname{LP}(\mathcal{A}, \mathcal{B}, \mathcal{C})$ and $\operatorname{LP}^{\prime}(\mathcal{A}, \mathcal{B}, \mathcal{C})$ are equivalent in expressive power and computational complexity. We use the first formulation in the fully general case of the formula bounding task Bound (Definition 6). For the kinds of inputs we get from Apriori and similar procedures, we actually have equalities for all input formulae, so we can use $\operatorname{LP}^{\prime}(\mathcal{A}, \mathcal{B}, \mathcal{C})$. Note that equalities $y=z$ can always be converted to the inequalities $y \leq z$ and $y \geq z$. We map the problem Bound into an instance of a linear programming problem $\operatorname{LP}(\mathcal{A}, \mathcal{B}, \mathcal{C})$ or $\operatorname{LP}^{\prime}(\mathcal{A}, \mathcal{B}, \mathcal{C})$ (depending on the kind of input). We talk about LP and inequalities in the following, but the case of $\mathrm{LP}^{\prime}$ and equalities is similar.

Assume now that $I$ is the solution of an instance of $\operatorname{Bound}(X, \Phi, f, \psi)$. By Lemma 1. we know that if the set $I$ is nonempty, it is a subinterval of $[0,1]$ (in rationals). Therefore, we proceed to compute its infimum; the case of the supremum is symmetric. Denote $n=|X|$, and denote the $2^{n}$ full clauses over $X$ by $\theta_{1}, \theta_{2}, \ldots, \theta_{2^{n}}$.

As input to Bound we have in effect a large set of inequalities that we will convert into one big matrix inequality $\mathcal{A} \boldsymbol{x} \leq \mathcal{B}$. The vector $\boldsymbol{x}$ will contain the unknowns: let $\boldsymbol{x}=\left(\left[\theta_{1}\right]\left[\theta_{2}\right] \ldots\left[\theta_{2^{n}}\right]\right)^{\mathrm{T}}$. Then, Proposition 2 yields for every formula $\phi \in \Phi$ a binary vector $\boldsymbol{k}=\left(k_{1} k_{2} \ldots k_{2^{n}}\right)$ such that the support of $\phi$ can be written as a matrix product, $[\phi]=\boldsymbol{k} \boldsymbol{x}$. Using this fact, we encode the constraint $[\phi] \in f(\phi)$ by adding into $\mathcal{A}$ two rows, $\boldsymbol{- k}$ and $\boldsymbol{k}$, and into $\mathcal{C}$ two numbers, $-a$ and $b$, where $[a, b]=f(\phi)$. Then any $\boldsymbol{x}$ satisfying $\mathcal{A} \boldsymbol{x} \leq \mathcal{B}$ must satisfy $a \leq \boldsymbol{k} \boldsymbol{x} \leq b$. Finally, as a necessary consistency constraint corresponding to the fact $[T]=1$, we add the rows $(-1-1 \ldots-1)$ and $(11 \ldots 1)$, and the numbers -1 and 1 . All in all, the dimensions of $\mathcal{A}$ will be $2(|\Phi|+1) \times 2^{n}$, and the dimensions of $\boldsymbol{x}$ and $\mathcal{B}$ will be $2(|\Phi|+1) \times 1$. Ways to reduce these dimensions will be discussed after Theorem 1 .

Having encoded all the constraints of the problem in $\mathcal{A}$ and $\mathcal{B}$, we now have to select $\mathcal{C}$ so that the solutions to the LP problem correspond to the supports of $\psi$. We once again invoke Proposition 2 to turn $[\psi]$ into a sum of supports of full clauses. Thus $\mathcal{C}$ will be a $0 / 1$ vector with $\mathcal{C} \boldsymbol{x}=[\psi]$, and minimizing $\mathcal{C} \boldsymbol{x}$ subject to the constraints gives the required infimum. When the bounds for the supports of input formulae are rational numbers, linear programming yields a rational value for the infimum, since for example the Simplex algorithm Kre93. $\S 21.3]$ uses only sums, differences, products and ratios to solve LP. Thus, the infimum corresponds to an assignment of nonnegative rational values to the supports of the full clauses, summing to 1 and obeying all the constraints of the original problem. Multiplying all the supports by the least common multiple of 
their denominators gives integer counts, whence a relation can be constructed. Thus the infimum is actually a minimum.

We have now proved the following theorem.

Theorem 1. The formula bounding task $\operatorname{Bound}(X, \Phi, f, \psi)$ can be reduced to the linear programming task $\operatorname{LP}(\mathcal{A}, \mathcal{B}, \mathcal{C})$. The matrix $\mathcal{A}$ will have $O(|\Phi|)$ rows and $2^{n}$ columns, and the vectors $\mathcal{B}$ and $\mathcal{C}$ will respectively have $O(|\Phi|)$ and $2^{n}$ elements, where $n=|X|$.

The output from our reduction has size $O\left(2^{n}|\Phi|\right)$, i.e., exponential in the number of attributes, where for the sake of simplicity we assume that all the numbers are represented using a fixed number of bits. Thus, a linear programming algorithm that requires polynomial time in the size of its input will take time that is polynomial in $\Phi$ but exponential in $n$. It would, therefore, be useful to diminish the exponential dependency on the number $n$ of attributes.

First, if $\Phi$ consists of frequent itemsets, we can restrict $X$ to only those attributes that appear in the query $\psi$. To see this, consider two full clauses $\theta$ and $\theta^{\prime}$ whose only difference is that $\theta$ has $A$ and $\theta^{\prime}$ has $\bar{A}$, where $A$ is an attribute that does not appear in $\psi$. The two coordinates in $\mathcal{C}$ corresponding to $\theta$ and $\theta^{\prime}$ will be equal, and thus only the sum $[\theta]+\left[\theta^{\prime}\right]$ will be relevant to the objective function $\mathcal{C} \boldsymbol{x}$. If a frequent set $\phi \in \Phi$ has different coordinates at the positions corresponding to the two full clauses, it must include $A$; then there is a frequent set $\phi^{\prime} \in \Phi$ that differs from $\phi$ only by excluding $A$. Thus in removing $\phi$ from $\Phi$ we lose no information relevant to $[\psi]$. Once all such frequent sets are gone, we can remove the attribute $A$ from $X$.

Second, we discuss whether using the family of all $2^{n}$ full clauses is necessary. One of the reasons we used full clauses was that they can be used to answer any support queries of Boolean formulae. However, many other families of formulae have this property. For example, Proposition 1 of [MT96] implies that the family of all conjunctions of atoms can be used to determine the supports of all Boolean formulae. Let us define a representation $\Theta$ over $X$ as a family of formulae such that the counts of all Boolean formulae over $X$ can be determined from the counts of the formulae in $\Theta$. In this context, we use integer $\operatorname{counts}^{\operatorname{count}_{r}}(\theta)=\sum_{t \in r}[\theta]_{t}$ instead of supports $[\theta]_{r}=\operatorname{count}_{r}(\theta) / \operatorname{count}_{r}(\top)$.

Any representation that works for all $r$ must have $2^{n}$ formulae. Indeed, given the counts corresponding to a representation, we can use Proposition 2 to form a linear system of equations from which the counts of full clauses can be solved. If there are fewer than $2^{n}$ equations, the system is underdetermined, and since all its factors are integers, it will have infinitely many integral solutions. It is therefore relatively easy to construct two relations with the same counts of all formulae of the supposed representation but different counts of some full clauses.

However, this does not rule out smaller representations that work for specific relations. When storing the counts of the conjunctions-of-atoms representation, we can leave out some counts that can be derived from others. If, e.g., there are no tuples satisfying the conjunction $A B$, we can leave out the count of $A B C$, and if the counts of $D$ and $D E$ are equal, we need store only one of the counts of $A D$ and $A D E$. Similar ideas have been studied in [ML98BBR00BR01. 
In our problem, we use fractional supports, not counts, which removes one degree of freedom. Since the supports of full clauses must add up to 1 , we can leave out one number from the full-clauses representation.

Third, in the case of $\mathrm{LP}^{\prime}$, where $\mathcal{A}$ is a $0 / 1$ matrix, we can often reduce the problem. If some row $\boldsymbol{a}_{i}$ of the matrix $\mathcal{A}$ is less than or equal to another row $\boldsymbol{a}_{j}$, we can replace $\boldsymbol{a}_{j}$ by $\boldsymbol{a}_{j}-\boldsymbol{a}_{i}$, while doing the corresponding replacement in $\mathcal{B}$. Sometimes this will result in a zero in $\mathcal{B}$; we can then deduce that several unknowns are zero and remove them. Even if this doesn't occur, the matrix becomes sparser, which helps some algorithms that solve linear programming problems.

We now continue the example bounding task of Table 2, We reduce the system depicted in the table to $\operatorname{LP}(\mathcal{A}, \mathcal{B}, \mathcal{C})$ with $\boldsymbol{x}=([A B][A \bar{B}][\bar{A} B][\bar{A} \bar{B}])^{\mathrm{T}}$. For exam-

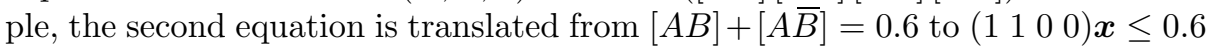
and $\left(\begin{array}{llll}-1 & -1 & 0 & 0\end{array}\right) \boldsymbol{x} \leq-0.6$. These inequalities form the third and fourth lines of $\mathcal{A}$ and $\mathcal{B}$ (see below). In this case, the first equation already forms the consistency constraint $\sum[\theta]=1$, so we need not add it now.

We obtain the values of $\boldsymbol{x}, \mathcal{A}$ and $\mathcal{B}$ listed in Table 3 , and $\mathcal{C}=\left(\begin{array}{llll}1 & 0 & 0 & 0\end{array}\right)$ (resp. $\left.\mathcal{C}=\left(\begin{array}{llll}-1 & 0 & 0 & 0\end{array}\right)\right)$ for finding the lower (resp. the upper) bound of $[A B]$. Solving these two LP problems gives the minimum 0.3 (with $\boldsymbol{x}=\left(\begin{array}{llll}0.3 & 0.3 & 0.4 & 0.0\end{array}\right)^{\mathrm{T}}$ ) and the maximum 0.5 (with $\left.\boldsymbol{x}=\left(\begin{array}{llll}0.5 & 0.1 & 0.2 & 0.2\end{array}\right)^{\mathrm{T}}\right)$. We can obtain actual relations by multiplying the values of $\boldsymbol{x}$ by 10 .

Table 3. The example bounding task converted into a linear program

$$
\boldsymbol{x}=\left(\begin{array}{l}
{[A B]} \\
{[A \bar{B}]} \\
{[\bar{A} B]} \\
{[\bar{A} \bar{B}]}
\end{array}\right), \quad \mathcal{A}=\left(\begin{array}{rrrr}
1 & 1 & 1 & 1 \\
-1 & -1 & -1 & -1 \\
1 & 1 & 0 & 0 \\
-1 & -1 & 0 & 0 \\
1 & 0 & 1 & 0 \\
-1 & 0 & -1 & 0 \\
1 & 0 & 0 & 0 \\
-1 & 0 & 0 & 0
\end{array}\right), \quad \mathcal{B}=\left(\begin{array}{r}
1 \\
-1 \\
0.6 \\
0.6 \\
-0.7 \\
0.5 \\
0
\end{array}\right),
$$

\section{Experiments}

We investigated the properties of the bounding procedure on two data sets. The first is connect-4 containing some game-state descriptions, the second is anpe, a database about unemployed people, set up by the French unemployment agency. We describe the specific properties of the data sets along with our results in Sections 4.2 and 4.3 .

We used as input to the bounding procedure different collections of frequent itemsets along with their supports $\mathrm{AMS}^{+96}$ MT96. As explained previously, an itemset is interpreted as the Boolean conjunction of items that it contains. Different collections of frequent itemsets correspond to different support thresholds, denoted by $\min _{\text {supp }}$.

In the implementation of the experiments, we used a less voluminous, although totally equivalent, representation of frequent itemsets, first described in BR01. Since this representation is smaller than all frequent itemsets, the resulting $\Phi$ contains fewer queries. The equivalence of representations guarantees 
that the same information can be inferred from it as from all frequent itemsets and their supports. We verified the equivalence by repeating some of the experiments using the ordinary frequent itemsets, and got exactly the same results.

\subsection{The Framework of the Experiments}

We can compute the support of a Boolean formula over an itemset $X$ exactly, if we know the supports of all subsets of $X$. The procedure for this computation in [MT96] is also applicable when we know the supports of frequent sets only, but then it will yield approximate bounds - it is sound but not complete. Thus, we test our new contribution using formulae over infrequent itemsets.

The protocol of the experiments can be simply put as following: we compare the average size of intervals inferred by Bound for 100 formulae, for which the combinatorial support-computing procedure of [MT96] is confronted with infrequent (thus missing) terms. The infrequent terms are due to the fact that the support threshold we use to mine frequent itemsets (considered further in the experiments with their corresponding supports as formulae with known supports) exceeds the support of some terms required by the procedure of MT96.

The detailed protocol is the following. For each of the two data sets, we selected $k=100$ random itemsets $X_{1}, \ldots, X_{k}$ that have 10 items each and whose supports do not exceed a predefined $\sigma_{\max }(10 \%$ for connect- 4 and $0.1 \%$ for anpe). To avoid selecting only itemsets with very low support, which typically account for the clobbering majority of all itemsets, we weighted the probability of selecting an itemset $X$ proportionally to its support $[X]$. Even then, most of the selected itemsets have low support compared to $\sigma_{\max }$ (on average, $2.26 \%$ for connect- 4 and $0.010 \%$ for anpe).

Based on these itemsets, we randomly drew $k$ Boolean formulae $\psi_{1}, \ldots, \psi_{k}$, one formula, $\psi_{i}$, over each $X_{i}$. To mimic formulae of interest in real life, for each $X_{i}$ we first selected a subset $Y_{i} \subseteq X_{i}$ of items, each item of $X_{i}$ with probability 0.7 . Then we defined $\psi_{i}$ as a disjunction of random full clauses over $Y_{i}$. We included each full clause $\theta$ in $\psi_{i}$ with probability $0.5-0.04 j$, where $j$ is the number of negative literals in $\theta$. Thus, we preferred clauses with more positive literals. For example, a clause with 10 negative literals had the probability of 0.1 to be included in $\psi_{i}$. Then we computed $\operatorname{Bound}\left(X_{i}, \Phi_{i}, f_{i}, \psi_{i}\right)$ where $\Phi_{i}$ consists of the precomputed frequent sets among the subsets of $X_{i}$, and $f_{i}$ assigns to each frequent set its known support. We report two scores, each an average over the 100 computations. Denoting the resulting lower and upper bounds by $L_{i}$ and $U_{i}$ for each computation, the first score is the average of $U_{i}-L_{i}$, the second the average of $\left(U_{i}-L_{i}\right) / U_{i}$, both averages over $i \in\{1, \ldots, 100\}$.

\subsection{Experiments with connect-4}

The connect-4 data set is very dense. It contains relatively small number of items (129) and rows (67557). 


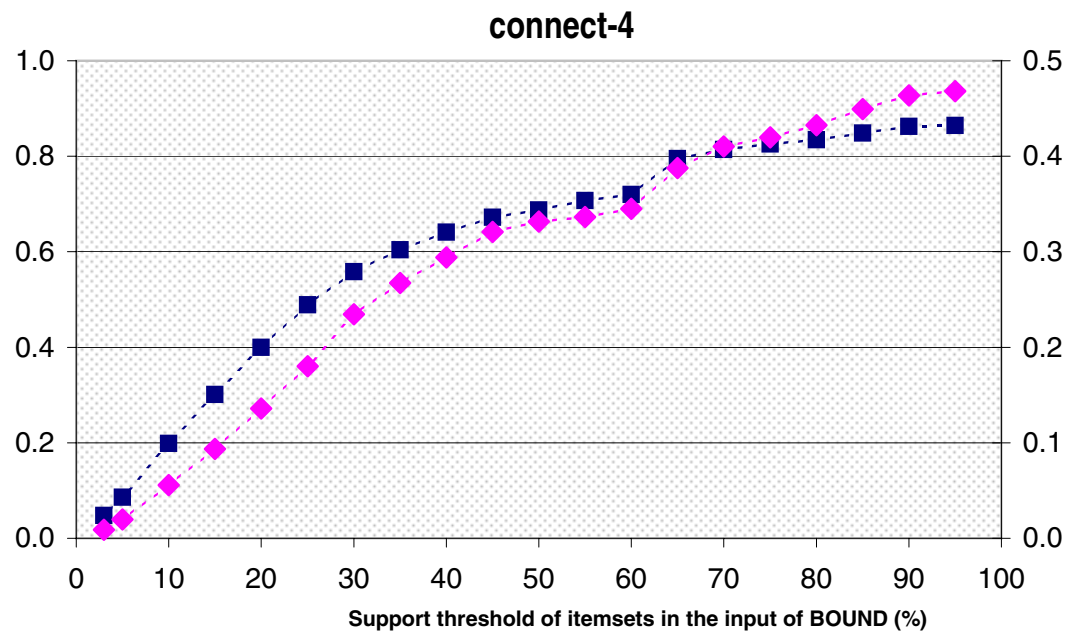

- - - - Relative interval size (left scale) - - - Absolute interval size (right scale)

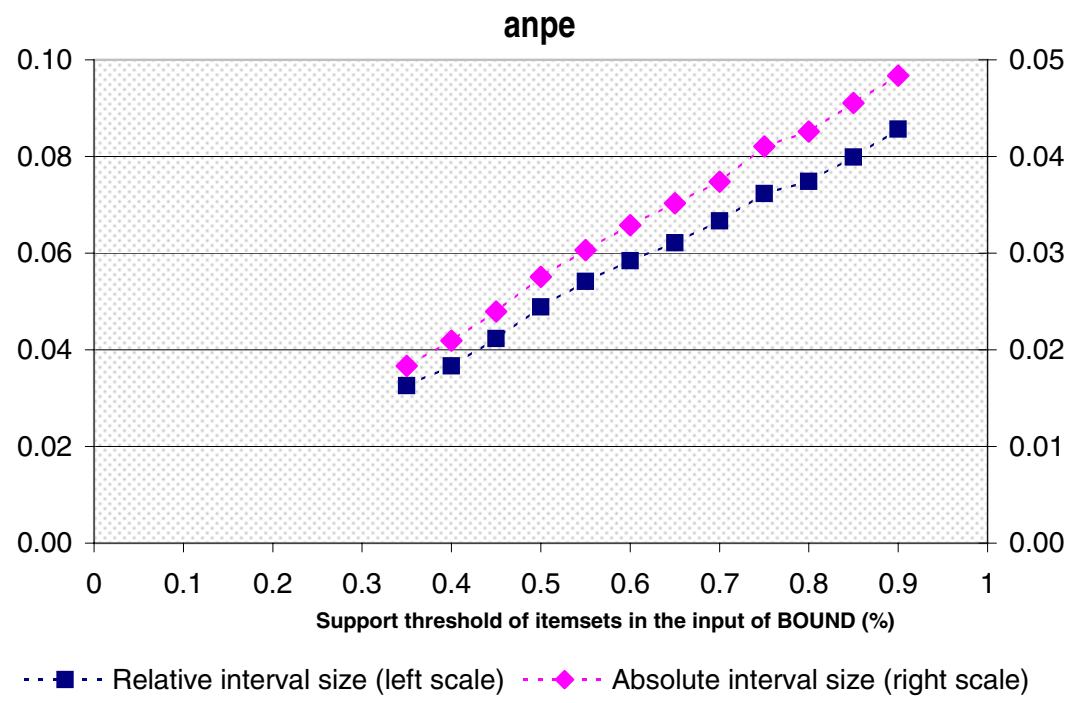

Fig. 1. Average interval size vs. input itemsets' support threshold produced by BOUND on the connect-4 and anpe data sets

In Figure 1 (top) we report the average size of the interval returned by the bounding procedure for different values of min $_{\text {supp }}$. The right-hand scale (diamonds) reports the difference between the ends of the interval, and the left-hand scale (squares) reports the ratio of this difference to the upper limit of the interval. As we can see, a lower min $_{\text {supp }}$ results in a better bounding precision. This is due to the increasing number of input itemsets, therefore a richer collection of information about the original data set. However, the cost associated with the 
computation and the use of these more voluminous collections of summaries also increases.

The support computation of [MT96] potentially involves an exponential number of terms for a single Boolean formula. Typically the computation will involve a significant part of the lattice of itemsets, subsets of the itemset on which we base our random formula. Since our random formulae are based on infrequent itemsets, many terms (often a majority) have unknown supports. However, the interval size we observe is of the same order of magnitude as the support threshold $\sigma$, which bounds the error of each unknown support. It seems that the unknown supports tend to cancel out, which appears to be a promising result.

Let us take an example. Consider the support threshold of $\sigma=15 \%$ and that the itemsets on which our random formulae are based have an average support of $2.26 \%$, and never have a support above $\sigma_{\max }=10 \%$. Take a single itemset and the corresponding formula; typically, a great number of the itemset's subsets are infrequent, each having support in the $[0, \sigma)$ range. When we compute the support of the formula as in [MT96, naturally most errors will cancel out, but one would not exepct the overall error to be in the $[0, \sigma)$ range; our method yields an average uncertainty of less than $10 \%$.

\subsection{Experiments with anpe}

The anpe data set is quite uncorrelated. With its 214 items and over 109000 rows, it is significantly larger than connect-4. Frequent set mining extracts relatively small collections, unless we set a very small min $_{\text {supp }}$. We chose to extract itemsets at these low thresholds. In Figure 1 (bottom) we report the average interval sizes. As previously, we relate the scores to different $\min _{\text {supp }}$.

The results look fairly similar to those of the previous experiment. In comparing the graphs it should be noted that the scaling of the axes is different: in this experiment, both the relative and the absolute errors are below 0.1 for all runs. In other words, this less dense data set allowed much greater precision in the resulting intervals.

\subsection{Observed Running Times}

In our experiments, we first gather summary query answers, to simulate either off-line or on-the-fly collecting of highly processed information. Then, we draw random formulae, as described in Section 4.1. For each random formula, we execute two steps: conversion into an $\operatorname{LP}^{\prime}(\mathcal{A}, \mathcal{B}, \mathcal{C})$ problem and solving it.

During the experiments, we observed that frequent itemset mining is the most expensive phase, despite the optimization of using an efficient condensed representation of the itemset collection described in BR01. For example, for the connect-4 data set and $\min _{\text {supp }}=5 \%$ it took more than 3000 seconds. Conversion to $\operatorname{LP}^{\prime}(\mathcal{A}, \mathcal{B}, \mathcal{C})$ took 4.78 seconds per formula on average, and solving $\operatorname{LP}^{\prime}(\mathcal{A}, \mathcal{B}, \mathcal{C})$ took only about 3.1 seconds per formula. Thus, the bounding procedure can be quite efficient in practice, after the frequent itemset mining has been performed. 


\section{Discussion and Future Work}

We have considered the problem of bounding the support of Boolean formulae when some aggregate information is available. We showed that the bounding problem can be reduced to a linear programming problem whose size can in the worst case be exponential in the number of attributes. While our result is foremost a theoretical one, we also gave empirical results showing that the bounding method can be effectively used to obtain additional information from frequent itemsets or other summaries.

We emphasize that our aim is to find exact bounds. Another approach would be to approximate the frequency of the query and give some kind of tail bounds for the error of the approximation. The most natural way would be to take a sample from the database and compute all queries on the sample; thus, instead of frequent sets, the sample would serve as the representation of the original data. This kind of a method has been used for computing frequent sets (see [Toi96]). A more sophisticated approximation can be based on frequent sets (or similar summaries) by building a probabilistic model over the variables occurring in the formula. A method using the maximum entropy principle is described in PMS00]. Like our solution, it suffers from exponential complexity in the number of variables occurring in the query.

Calders and Goethals Cal02 CG02 have studied a similar problem. They have derived deduction rules for bounding the support of an itemset given the exact supports of all its proper subsets. While the rules are sound and complete for that task, they don't solve our more general problem. In particular, these rules are not applicable when the supports of some subsets are unknown. Thus they cannot derive directly the support of a derivable itemset, but must first bound recursively the supports of all its proper subsets. They deal only with itemsets, i.e., conjunctions of attributes, not arbitrary formulae. The full set of rules is exponentially large, although Calders and Goethals give experimental evidence that a small subset of the rules suffices to give a reasonably good result.

Several open problems remain. One area is obtaining a faster method for the inference problem. With large, redundant summaries such as frequent itemsets, the solution by linear programming is quite slow, and it is in many cases outperformed by the simple "scan the database once and count" method. The method could, however, be useful in cases where the data set is not available or where the set of queries $\Phi$ (corresponding to known supports) carries a lot of information condensed in well chosen summaries, orders of magnitude smaller than the data set itself. Thus, the following fundamental issue is interesting.

Problem 1. Given a relation $r$, an amount $Z$ of storage, and a class of queries $\Psi$ that we wish to perform on $r$, what should we store in $Z$ (which presumably cannot hold all of $r$ ) in order to most effectively answer the queries in $\Psi$ ?

Frequent sets are typically redundant collections, and thus are not optimal. In fact, in our experiments we used the smaller collection of disjunction-free sets BR01, and further gains could be obtained using the Calders-Goethals rules [CG02. Another interesting representation is the AD-tree ML98. In gen- 
eral, if we store in $Z$ the answers to some Boolean queries $\phi_{1}, \phi_{2}, \ldots, \phi_{N}$, the linear programming approach shows the limits of what we can reconstruct. Perhaps a suitable set of formulae would allow an analytical solution, possibly only approximate, of the linear program. The problem of computing frequent sets from data has been extensively studied, and they were used in [MT96, which formed the starting point for our research. But the linear programming framework does not depend on them - it can be used with supports of any formulae.

Another interesting issue is how to relax (if possible) the requirements of Definition [6] if the complete procedure is too slow. We do not want to give unsound answers, but too wide intervals are not necessarily harmful. The simplest incomplete and sound algorithm "return the interval $[0,1]$ " is not useful, but we suspect there might be a reasonably fast compromise between it and the complete linear programming approach.

Problem 2. How close to completeness can a polynomial-time (or linear-time, or randomized polynomial-time) sound solution to Bound come?

\section{Acknowledgements}

Part of the work was done when Artur Bykowski was visiting the Laboratory of Computer and Information Science at Helsinki University of Technology. The connect-4 data set was provided by researchers at the IBM Almaden research center, and the anpe data set was preprocessed and anonymized by Christophe Rigotti at INSA-Lyon. Professor Heikki Mannila presented to us the question about support inference and gave useful comments on an earlier version of this manuscript.

\section{References}

[AMS ${ }^{+}$96] Rakesh Agrawal, Heikki Mannila, Ramakrishnan Srikant, Hannu Toivonen, and A. Inkeri Verkamo. Fast discovery of association rules. In Usama M. Fayyad, Gregory Piatetsky-Shapiro, Padhraic Smyth, and Ramasamy Uthurusamy, editors, Advances in Knowledge Discovery and Data Mining, chapter 12, pages 307-328. AAAI Press, 1996.

[BBR00] Jean-François Boulicaut, Artur Bykowski, and Christophe Rigotti. Approximation of frequency queries by means of free-sets. In $P K D D$ '00, Lecture Notes in Computer Science, Vol. 1910, pages 75-85. Springer, 2000.

[BR01] Artur Bykowski and Christophe Rigotti. A condensed representation to find frequent patterns. In Proc. of the 20th ACM SIGACT-SIGMODSIGART Symposium on Principles of Database Systems (PODS'01), Santa Barbara, CA, USA, May 2001. ACM.

[Cal02] Toon Calders. Deducing bounds on the frequency of itemsets. In EDBT 2002 Workshop on Database Technologies for Data Mining, 2002.

[CG02] Toon Calders and Bart Goethals. Mining all non-derivable frequent itemsets. In Tapio Elomaa, Heikki Mannila, and Hannu Toivonen, editors, Proceedings of the 6th European Conference on Principles of Data Mining and Knowledge Discovery, volume 2431 of Lecture Notes in Computer Science, pages 74-85. Springer-Verlag, 2002. 
[GZ01] Karam Gouda and Mohammed Javeed Zaki. Efficiently mining maximal frequent itemsets. In Proc. of the 2001 IEEE International Conference on Data Mining (ICDM'01), pages 163-170, San Jose, California, USA, 2001.

[HPY00] Jiawei Han, Jian Pei, and Yiwen Yin. Mining frequent patterns without candidate generation. In Weidong Chen, Jeffrey Naughton, and Philip A. Bernstein, editors, 2000 ACM SIGMOD Intl. Conference on Management of Data, pages 1-12. ACM Press, May 2000.

[Kar84] N. Karmarkar. A new polynomial-time algorithm for linear programming. In Proceedings of the sixteenth annual ACM symposium on Theory of computing, pages 302-311, 1984.

[Kre93] Erwin Kreyszig. Advanced Engineering Mathematics. John Wiley Inc., seventh edition, 1993.

[ML98] Andrew Moore and Mary Soon Lee. Cached sufficient statistics for efficient machine learning with large datasets. Journal of Artificial Intelligence Research, 8:67-91, 1998.

[MSW96] Jiří Matoušek, Micha Sharir, and Emo Welzl. A subexponential bound for linear programming. Algorithmica, 16(4/5):498-516, 1996.

[MT96] Heikki Mannila and Hannu Toivonen. Multiple uses of frequent sets and condensed representations: Extended abstract. In Proc. of the 2nd International Conference on Knowledge Discovery and Data Mining (KDD'96), pages 189-194, Portland, Oregon, USA, August 1996. AAAI Press.

[MTV97] Heikki Mannila, Hannu Toivonen, and A. Inkeri Verkamo. Discovery of frequent episodes in event sequences. Data Mining and Knowledge Discovery, 1(3):259-289, 1997.

[PBTL99] Nicolas Pasquier, Yves Bastide, Rafik Taouil, and Lotfi Lakhal. Efficient mining of association rules using closed itemset lattices. Information Systems, 24(1):25-46, 1999.

[PMS00] Dmitry Pavlov, Heikki Mannila, and Padhraic Smyth. Probabilistic models for query approximation with large sparse binary datasets. In Proc. of the 16th Conference in Uncertainty in Artificial Intelligence (UAI'00), Stanford, California, USA, 2000.

[Toi96] Hannu Toivonen. Sampling large databases for association rules. In Proc. of the 22th International Conference on Very Large Data Bases (VLDB'96), pages 134-145, Mumbai (Bombay), India, 1996.

[ZRL97] Tian Zhang, Raghu Ramakrishnan, and Miron Livny. BIRCH: A new data clustering algorithm and its applications. Data Mining and Knowledge Discovery, 1(2):141-182, 1997. 